\title{
Removing Line Scratches in Digital Image Sequences by Fusion Techniques
}

\author{
Giuliano Laccetti ${ }^{1}$, Lucia Maddalena ${ }^{2}$, and Alfredo Petrosino ${ }^{3}$ \\ 1 University of Naples , "Federico II" Via Cintia, 80126 Naples, Italy \\ giuliano.laccetti@dma. unina.it \\ 2 Italian National Research Council, ICAR, Via P. Castellino 111, 80131 Naples, Italy \\ lucia.maddalena@na.icar.cnr.it \\ 3 University of Naples "Parthenope", Via A. De Gasperi 5, 80133 Naples, Italy \\ alfredo.petrosino@uniparthenope.it
}

\begin{abstract}
Many algorithms have been proposed in literature for digital film restoration; unfortunately, none of them ensures a perfect restoration whichever is the image sequence to be restored. Here, we propose an approach to digital scratch restoration based on image fusion techniques for combining relatively well settled distinct techniques. Qualitative results are deeply investigated for several real image sequences.
\end{abstract}

\section{Introduction}

With the recent advent of digital technologies, and the ever increasing need for speed and storage, the removal of occluded or missing parts in images and movies is a more and more widespread problem. The problem can occur in several multimedia applications, such as wireless communication and digital image sequence restoration. Several classes of defects can be distinguished that affect movies, and many algorithms have been proposed in literature for their restoration; unfortunately, none of them ensures a perfect restoration whichever is the image sequence to be restored.

A sufficiently general model of degraded video signal is the following for a pixel location $\mathbf{p}=(x, y)$ :

$$
I(\mathbf{p}, t)=(1-b(\mathbf{p}, t)) I^{*}(\mathbf{p}, t)+b(\mathbf{p}, t) c(\mathbf{p}, t)+\eta(\mathbf{p}, t),
$$

where $I(\mathbf{p}, t)$ is the corrupted signal at spatial position $\mathbf{p}$ in frame $t, b(\mathbf{p}, t) \in$ $\{0,1\}$ is a binary mask indicating the points belonging to missing parts of the degraded video, $I^{*}$ is the ideal uncorrupted image. The (more or less constant) intensity values at the corrupted spatial locations are given by $c(\mathbf{p}, t)$. Though noise is not considered to be the dominant degrading factor in the defect domain, it is still included in (1) as the term $\eta(\mathbf{p}, t)$.

In the present paper, we focus on the class of scratch defects, intended as long and thin vertical scratches that affect several subsequent images of a sequence, due to the abrasion of the film by dust particles in the slippage mechanisms used for the development, projection and duplication of the film. 
Commonly, scratch restoration is a two-step procedure. In the first step the scratches need to be detected, i.e., an estimate for the mask $b(\mathbf{p}, t)$ is made (detection step). In the second step the values of $I^{*}$ inside the scratch, possibly starting from information about $c(\mathbf{p}, t)$, are estimated (removal step). As usual, we consider scratch reduction as a problem of detection and removal of missing data, i.e. we suppose that any information $c(\mathbf{p}, t)$ has been lost within the scratch.

Several scratch restoration methods are reported in literature (see for instance refs. 1] through [13]). As expected, advantages and disadvantages characterize each scratch detection and removal technique, and any of them could be said to win the competition. A way to deal with this kind of problems is to adopt fusion techniques (see for instance 1415]): input images which provide alternative and complementary "views" and "characteristics" of a given area are "fused" into a single image. Fusion techniques should ensure that all the important visual information found in input images is transferred into the fused output image, without the introduction of artifact distortion effects. In this sense machine vision systems can be organized as a set of separated visual modules that act as virtual sensors. In this paper, the term visual module indicates an algorithm that extracts information of some specific and descriptable kind from a numerical image. For what concerns digital scratch restoration, a fusion technique may be applied both to the detection stage and to the removal stage: in both cases it takes into account already existing promising algorithms and suitably combines the obtained results in order to provide a restored sequence as similar as possible to the original uncorrupted sequence. As we show, the results produced by the proposed approach upon different damaged movies greatly enhance those produced by each considered approach. The experimental results reported in literature showed that the accuracy provided by the combination of an ensemble of visual modules can outperform the accuracy of the best single visual module.

The contents of this paper are as follows: Section 2 outlines the proposed compound algorithm for scratch restoration, based on scratch detection and removal modules and suitable detection and removal fusion strategies. Section 3 describes the qualitative results achieved by the proposed fusion approach tested on real video sequences. Conclusions are reported in Section 4.

\section{Proposed Algorithm}

The considered algorithm for scratch restoration in image sequences is based on an approach that takes into account already existing promising algorithms and suitably combines the obtained results in order to provide a restored sequence as similar as possible to the original uncorrupted sequence [16. The basic idea of the compound algorithm consists, for each sequence frame, in:

1. applying a set of $d$ existing scratch detection algorithms;

2. combining obtained scratch masks $B^{j}, j=1, \ldots, d$, to produce the final scratch mask $B^{C}$; 
3. applying a set of $r$ existing scratch removal algorithms using scratch mask $B^{C}$;

4. combining obtained restored images $R^{j}, j=1, \ldots, r$, to produce the final restored image $R^{C}$.

For the implementation of the compound algorithm we have considered as underlying restoration modules three detection algorithms presented in [789] and two removal algorithms presented in 311. Moreover, for the combination of results we used fusion techniques which allow to exploit the performance of underlying algorithms while reducing their drawbacks 16, briefly described in the following. With such techniques input images, providing alternative and complementary views of a given region, are fused into a single image, in such a way that all the relevant content of input images is transferred to the fused output image, without introducing distortions.

\subsection{Detection Fusion Strategies}

The main goal of using more than one detection visual module is to make up for deficiencies in the individual modules, while retaining their features, thus achieving a better overall detection result than each single module could provide. In this case the combination should be made among scratch masks $B^{j}$ produced by the detection modules, $j=1, \ldots, d$ (in our case $d=3$ ). Here, two different combining methods or aggregation operators are adopted; supposing, for simplicity, that damaged images are affected by just one scratch, their result $B^{C}$ is given by:

- Union aggregation operator: $B^{C}=\cup\left\{B^{j}: j=1, \ldots, d\right\}$ such that $B^{C}(x, y)=$ $\max _{j}\left\{B^{j}(x, y)\right\}, \forall(x, y)$;

- Maximum Covering (MC) aggregation operator): $B^{C}=M C\left\{B^{j}: j=\right.$ $1, \ldots, d\}$ such that, for all $y=0, \ldots, M-1$ ( $M=$ number of image rows),

$$
B^{C}(x, y)=\left\{\begin{array}{l}
1 \text { if } x \in\left[x^{\text {mean }}-W, x^{\text {mean }}+W\right] \\
0 \text { otherwise }
\end{array},\right.
$$

where $W=\max \left\{\left|x^{\text {mean }}-x^{\text {min }}\right|,\left|x^{\text {mean }}-x^{\text {max }}\right|\right\}, x^{\text {mean }}=\operatorname{mean}(X), x^{\text {min }}=$ $\min (X), x^{\max }=\max (X), X=\left\{x: \cap_{j} B^{j}(x, y)=\min _{j}\left\{B^{j}(x, y)\right\}=1, \forall y\right\}$.

\subsection{Removal Fusion Strategies}

The problem here can be stated as follows: given $r$ images representing heterogeneous data on the observed phenomenon, take a decision $D_{i}$ on an element $(x, y)$ where $D_{i}$ belongs to a decision space $D$. In image fusion the information relating $(x, y)$ to each possible decision $D_{i}$ is represented as a number $M_{i}^{j}$, where $j$ indexes the decision making module having different properties and different meanings depending on the mathematical framework. Given images $R^{j}$ obtained by removal module $j, j=1, \ldots, r$ (in our case $r=2$ ), if we assume 
that $M_{i}^{j}(x, y)=R^{j}(x, y)$, with $(x, y)$ in the scratch domain, represents the probability degree to which the pixel $(x, y)$ could be seen as "restored" ( $i$ indexes the values of this appearance), we can claim all the advantages of the Bayesian framework relying in the variety of combination operators. Here we adopt the averaging aggregation operator, known in the Bayesian framework as the Basic Ensemble Method [17. (see also [1819) for combining different classification modules, which has been demonstrated to significantly improve the classification performance of each single module: $R^{C}=B E M\left\{R^{j}: j=1, \ldots, r\right\}$, such that $R^{C}(x, y)=\frac{1}{r} \sum_{j=1}^{r} R^{j}(x, y) \forall(x, y)$.

\section{Qualitative Results}

\subsection{Test Data}

Detection and removal algorithms here presented have been tested on several real images. Moreover, the considered algorithms have been tested also on artificially corrupted images. Specifically we considered $K=20$ uncorrupted original $\mathrm{B} / \mathrm{W}$ images $I^{k}, k=1, \ldots, K$, each of size $N \times M=256 \times 256$, and the corresponding images with an artificial scratch of odd width $w$, denoted as $I^{k, w}, k=1, \ldots, K ; w=3,5, \ldots, 19$, obtained as:

$$
I^{k, w}(x, y)=\left\{\begin{array}{cl}
255 & \text { if }(x, y) \in \Omega_{w}-\partial \Omega_{w} \\
200 & \text { if }(x, y) \in \partial \Omega_{w} \\
I^{k}(x, y) & \text { otherwise }
\end{array}\right.
$$

where $\Omega_{w}$ denotes the scratch domain, that is the rectangular subset of the image domain of size $w \times M$ having as first column the center column $N / 2$ of the image: $\Omega_{w}=\left\{(x, y): x=\frac{N}{2}, \ldots, \frac{N}{2}+w-1 ; y=0, \ldots, M-1\right\}$, and $\partial \Omega_{w}$ denotes its border.

\subsection{Detection Results}

The accuracy of the result of the detection algorithms taken into account (not reported here for space constraints) is quite high. Nonetheless, the aggregated masks seem more appropriate for the successive removal phase. Comparing the two aggregated masks, the union aggregation operator allows to consider all details captured by the different detection algorithms, while retaining the minimum support of the mask; the MC aggregation operator, instead, leads to a mask that appears unnatural for real images (being perfectly rectangular).

In order to obtain an objective estimate of detection algorithms, for each mask $B^{k}$ computed with anyone of the described detection algorithms for the artificially scratched image $I^{k, w}$ described in eqn. (2) we count:

$$
C^{k, w}=\operatorname{card}\left\{(x, y):(x, y) \in \Omega_{w}, B^{k}(x, y)=1\right\} \text {, number of correct detections }
$$

(pixels of the scratch that are included in the computed scratch mask); 
Table 1. Correct detection rates $r_{C}$ and false alarm rates $r_{F}$ for the detection algorithms applied to images of eqn. (2) varying the scratch width $w$.

\begin{tabular}{lcccccccc}
\hline & $w=3$ & $w=5$ & $w=7$ & \multicolumn{2}{c}{$w=9$} \\
\hline & $r_{C}$ & $r_{F}$ & $r_{C}$ & $r_{F}$ & $r_{C}$ & $r_{F}$ & $r_{C}$ & $r_{F}$ \\
\hline Detection alg. 1 & 0.9984 & 0.0000 & 0.9991 & 0.0000 & 0.9991 & 0.0000 & 0.9991 & 0.0000 \\
Detection alg. 2 & 0.9684 & 0.0006 & 0.9565 & 0.0015 & 0.9697 & 0.0017 & 0.9765 & 0.0017 \\
Detection alg. 3 & 0.9984 & 0.0000 & 0.9991 & 0.0000 & 0.9996 & 0.0000 & 0.9995 & 0.0000 \\
Union fusion aggr. op. 0.9984 & 0.0006 & 0.9991 & 0.0015 & 0.9996 & 0.0017 & 0.9995 & 0.0017 \\
MC fusion aggr. op. & 1.0000 & 0.0012 & 1.0000 & 0.0024 & 1.0000 & 0.0024 & 1.0000 & 0.0028 \\
\hline
\end{tabular}

$F^{k, w}=\operatorname{card}\left\{(x, y):(x, y) \notin \Omega_{w}, B^{k}(x, y)=1\right\}$, number of false alarms (pixels not belonging to the scratch that are included in the computed scratch mask),

and their mean values $C^{w}$ and $F^{w}$ over the $K$ images of eqn. (2): $C^{w}=$ $\frac{1}{K} \sum_{k=1}^{K} C^{k, w} ; F^{w}=\frac{1}{K} \sum_{k=1}^{K} F^{k, w}$. Given the scratch width $w$, the measures adopted for the objective estimation of the detection algorithms are:

- correct detection rate $r_{C}=\frac{C^{w}}{w \times M}, w \times M$ being the number of corrupted pixels (i.e. the dimension of the set $\Omega_{w}$ ). Such measure gives values in $[0,1]$; the higher the value of $r_{C}$, the better the detection result;

- false alarm rate $r_{F}=\frac{F^{w}}{N \times M-w \times M}$. Such measure gives values in $[0,1]$; the lower the value of $r_{F}$, the better the detection result.

Values for $r_{C}$ and $r_{F}$ obtained with all the described detection algorithms are reported in Table1, varying the scratch width $w=3,5,7$, and 9. Here we can observe that $r_{C}$ values are generally very close to 1 for all detection algorithms and that only few false alarms are generated. Specifically, we observe that the union fusion strategy reaches the best $r_{C}$ and the worst $r_{F}$ values achieved by the underlying detection algorithms; the MC fusion strategy reaches the best $r_{C}$ values attainable $\left(r_{C}=1\right)$, but $r_{F}$ values worse than any other algorithm.

\subsection{Removal Results}

The results of scratch removal (not reported here for space constraints) show that, even though the removal algorithms taken into account perform quite well, their reconstruction accuracy can be enhanced; the aggregated results, instead, tend to smooth the inaccuracies, still retaining the good performance of the considered algorithms.

In order to obtain objective measures of the removal algorithms accuracy, we tested them on the artificially scratched images $I^{k, w}$ described in eqn. (2). Given the scratch width $w$, let be, for $k=1, \ldots, K, \mathbf{o}_{k}$ the vector of dimension $\operatorname{card}\left(\Omega_{w}\right) \times 1$ obtained scanning row by row the subimage of $I^{k}$ for pixels in $\Omega_{w}$, and $\mathbf{r}_{k}$ the vector of dimension $\operatorname{card}\left(\Omega_{w}\right) \times 1$ obtained scanning row by row the subimage of $R^{k}$ for pixels in $\Omega_{w}$. We consider the following objective measures: 


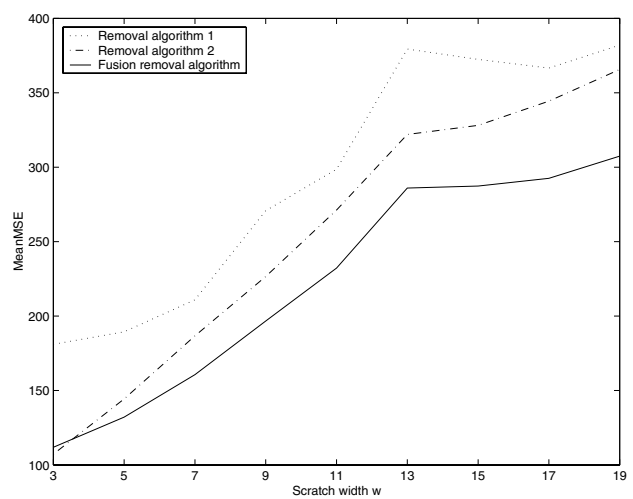

(a)

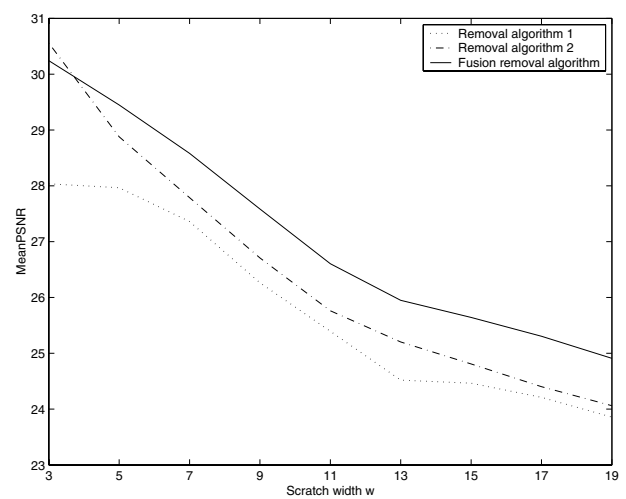

(b)

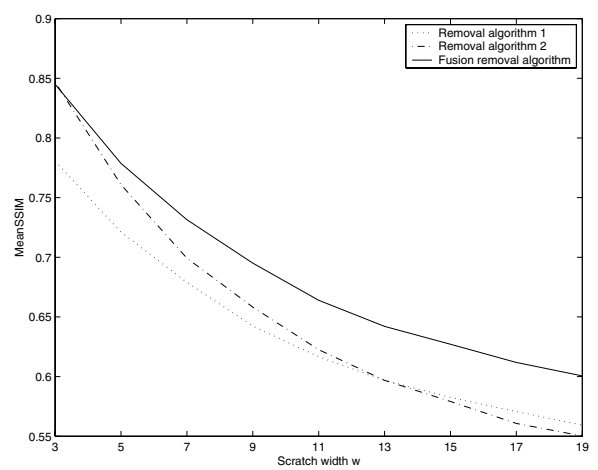

(c)

Fig. 1. Accuracy measures of the removal algorithms applied to images described in eqn. (2): (a) MeanMSE, (b) MeanPSNR, (c) MeanSSIM

- MeanMSE $=\frac{1}{K} \sum_{k=1}^{K} \frac{1}{w * M}\left\|\mathbf{o}_{k}-\mathbf{r}_{k}\right\|^{2}$, mean, over the $K$ restored images, of the Mean Square Error (MSE) between the original and the restored images. Such measure gives a nonnegative value; the smaller the value of MeanMSE, the better the restoration result;

- MeanPSNR $=\frac{1}{K} \sum_{k=1}^{K}\left(10 * \log _{10}\left(\frac{255^{2}}{\frac{1}{w * M}\left\|\mathbf{o}_{k}-\mathbf{r}_{k}\right\|^{2}}\right)\right)$, mean, over the $K$ restored images, of the Peak-to-Noise-Ratio between the original and the restored images obtained considering the MSE. Such measure gives a nonnegative value; the higher the value of $\operatorname{MeanPSNR}$, the better the restoration result;

- MeanSSIM $=\frac{1}{K} \sum_{k=1}^{K}\left(\frac{\left(2 * \mu_{\mathbf{o}_{k}} * \mu_{\mathbf{r}_{k}}+C_{1}\right)\left(2 * \sigma_{\mathbf{o}_{k} \mathbf{r}_{k}}+C_{2}\right)}{\left(\mu_{\mathbf{o}_{k}}^{2}+\mu_{\mathbf{r}_{k}}^{2}+C_{1}\right)\left(\sigma_{\mathbf{o}_{k}}^{2}+\sigma_{\mathbf{r}_{k}}^{2}+C_{2}\right)}\right)$, mean, over the $K$ restored images, of the Structural Similarity Index [20] applied to the 
original and the restored images, where $C_{1}=\left(K_{1} * L\right)^{2}, C_{2}=\left(K_{2} * L\right)^{2}, K_{1}=$ $0.01, K_{2}=0.03$, and $L=255$. Such measure gives values in $[0,1]$; the higher the value of MeanSSIM, the better the restoration result.

Results obtained with the described measures varying the scratch width $w$ are reported in Fig. 1, It can be observed that MeanMSE values obtained with the fusion removal algorithm are always lower than those obtained with the two removal algorithms and that MeanPSNR and MeanSSIM values obtained with the fusion removal algorithm are always higher than those obtained with the two removal algorithms. Moreover, for each removal method, results obtained with all the considered measures show lower accuracy increasing the scratch width, in accordance with the increasing reconstruction difficulty as the reconstruction area widens.

In summary, we can state that the considered measures indicate the fusion method as the most accurate among the considered removal methods.

\section{Conclusions and Ongoing Work}

This paper described an innovative algorithm for line scratch restoration based on data fusion techniques to detect and restore scratches in digital corrupted images. The described compound algorithm has been tested on several corrupted and artificially corrupted real images in order to analyze the results accuracy in terms of objective measures, showing that the compound algorithm outperforms the underlying restoration methods.

Ongoing researches deal with the analysis and adoption of alternative aggregation operators, like the Ordered Weighted Aggregation operators due to Yager [21] or the Non-liner Generalized Ensemble Method as introduced in 22].

\section{References}

1. Acton, S.T., Mukherjee, D.P., Havlicek, J.P., Bovik, A.C.: Oriented Texture Completion by AM-FM Reaction-Diffusion. IEEE Transactions on Image Processing 10 (2001) 885-896

2. Bertalmio, M., Sapiro, G., Caselles, V., Ballester, C.: Image Inpainting. Computer Graphics (2000) 417-424

3. Bornard, R., Lecan, E., Laborelli, L., Chenot, J.-H.: Missing Data Correction in Still Images and Image Sequences. In Proc. ACM Multimedia 2002, Juan-les-Pins, France (2002) 355-361

4. Chan, T.F., Shen, J.: Mathematical Models for Local Non-Texture Inpaintings. UCLA CAM Report n. 00-11 (2000)

5. Decenciere Ferrandiere, E.: Restauration Automatique de Films Anciens. PhD Thesis, Ecole Nationale Superieure des Mines de Paris (1997)

6. Isgró, F., Tegolo, D.: A distributed genetic algorithm for restoration of vertical line scratches. Accepted for publication in Parallel Computing

7. Joyeux, L., Boukir, S., Besserer, B.: Tracking and MAP Reconstruction of Line Scratches in Degraded Motion Pictures. Machine Vision and Applications 13 (2002) $119-128$ 
8. Kao, O., Engehausen, J.: Scratch Removal in Digitised Film Sequences. In Proc. International Conference on Imaging Science, Systems, and Technology (CISST) (2000) $171-179$

9. Kokaram, A.C.: Motion Picture Restoration: Digital Algorithms for Artefacts Suppression in Archived Film and Video. Springer-Verlag (1998)

10. Machì A., Collura, F., Nicotra, F.: Detection of Irregular Linear Scratches in Aged Motion Picture Frames and Restoration using Adaptive Masks. In Proc. IASTED Int. Conf. SIP02, Kawai, Usa (2002) 254-259

11. Maddalena, L.: Efficient Methods for Scratch Removal in Image Sequences. In Proc. 11th International Conference on Image Analysis and Processing (ICIAP2001), IEEE Computer Society (2001) 547-552

12. Morris, R.D.: Image Sequence Restoration Using Gibbs Distributions. PhD Thesis, University of Cambridge (1995)

13. Saito, T., Komatsu, T., Ohuchi, T., Seto, T.: Image Processing for Restoration of Heavily-Corrupted Old Film Sequences. In Proc. ICPR'00, IEEE, Barcellona (2000) 3017-3020

14. Bloch, I.: Information Combination Operators for Data Fusion: A Comparative Review with Classification. IEEE Transactions on Systems, Man, Cybernetics 26 (1996) 52-67

15. Ho, T.K., Hull, J.J., Srihari, S.N.: Decision Combination in Multiple Classifier Systems. IEEE Transactions on Pattern Analysis and Machine Intelligence 18 (1994) 66-75.

16. Laccetti, G., Maddalena, L., Petrosino, A.: Parallel/Distributed Film Line Scratch Restoration by Fusion Techniques. In: Lagan, A. et al. (eds.): Computational Science and Its Applications - ICCSA 2004, Lecture Notes in Computer Science, Vol. 3044. Springer (2004) 524-534

17. Perrone, M.P., Cooper, L.N.: When networks disagree: Ensemble method for neural networks. In Mammone, R.J. (ed.): Artificial Neural Networks for Speech and Vision. Chapman \& Hall, New York (1993) 126-142

18. Roli, F.: Linear Combiners for Fusion of Pattern Classifiers. Int. School on Neural Nets, E.R. Caianiello, 7th Course on Ensemble Methods for Learning Machines, Vietri sul Mare, Italy (2002)

19. Fumera, G., Roli, F.: A Theoretical and Experimental Analysis of Linear Combiners for Multiple Classifier Systems. IEEE Transactions on Pattern Analysis and Machine Intelligence. (in press)

20. Wang, Z., Lu, L., Bovik, A.C.: Video Quality Assessment Based on Structural Distortion Measurement. Signal Processing: Image Communication 19 (2004) 121132

21. Yager, R.R., Kacprzyk, J.: The Ordered Weighted Averaging Operation: Theory, Methodology and Applications. Kluwer: Norwell, MA (1997)

22. Ceccarelli, M., Petrosino, A.: Multifeature adaptive classifiers for SAR image segmentation. Neurocomputing 14 (1997) 345-363 